\title{
Efeitos da Utilização de Exergames na Reabilitação de Pacientes com Síndrome Metabólica
}

\author{
Effects of Using Exergames on Rehabilitation of Patients with Metabolic Syndrome
}

\author{
Karina Langone Vieira ${ }^{1}$ \\ César Augusto OteroVaghetti ${ }^{2}$ \\ André de Oliveira Teixeira ${ }^{3}$
}

\section{RESUMO}

Objetivo: Investigar os efeitos do game YourShape: Fitness Evolved, do console Xbox 360 sobre a capacidade física, as variáveis hemodinâmicas, a qualidade de vida e a percepção subjetiva de esforço. Metodologia: Participaram da pesquisa 15 indivíduos adultos, com afeç̧ões cardiometabólicas, tais como diabetes mellitus, hipertensão, insuficiência cardíaca, hipotireoidismo e dislipidemia, com idade superior a 18 anos. As sessões de treinamento tinham duração de 60 minutos, perfazendo um total de 12 sessões, três vezes por semana, sendo iniciadas com uma caminhada na esteira, durante 30 minutos e logo após os exercícios em ambiente virtual no EXG, também por 30 minutos. Resultados: Foram encontradas diferenças estatisticamente significativas entre a primeira e a última sessão de treinamento, caminhada e EXG (YourShape: Fitness Evolved), para as variáveis circunferência de cintura, percepção subjetiva de esforço, taxa glicêmica e alguns aspectos do questionário de qualidade de vida como a capacidade funcional, os aspectos físicos, a dor, a vitalidade e a saúde mental. Além disso, a taxa glicêmica também apresentou uma diferença significativa entre o pré e o pós-intervenção, em todas as sessões. Conclusão: Os resultados evidenciam o caráter inovador da realidade virtual, especialmente os exergames, devido as suas características motivacionais e o seu potencial para a prescrição de exercício físico, assim como o baixo custo e a usabilidade para utilização em hospitais.

\section{DESCRITORES}

Realidade Virtual. Síndrome Versus Metabólica. Reabilitação.

\begin{abstract}
Objective: To investigate the effects of the game YourShape: Fitness Evolved, from the Xbox 360 console on the physical capabilities, hemodynamic variables, quality of life and subjective perception of effort. Methodology: The study included 15 adult individuals with cardiometabolic disorders, such as diabetes mellitus, hypertension, heart failure, hypothyroidism and dyslipidemia, aged over 18 years. The training sessions lasted 60 minutes, in a total of 12 sessions, three times a week, starting with walking on the treadmill, for 30 minutes, followed by the exercises in a virtual environment on the exergame, also for 30 minutes. Results: There were statistically significant differences between the first and last training session, walking and EXG (YourShape: Fitness Evolved), for the variables waist circumference, subjective perception of effort, glycemic rate and some aspects of the quality-of-life questionnaire such as functional capacity, physical aspects, pain, vitality and mental health. In addition, the glycemic rate also showed a significant difference between pre-test and post-test in all sessions. Conclusion: the results show the innovative character of Virtual Reality, especially the Exergames, due to its motivational characteristics, and its potential for the prescription of physical exercise, as well as low cost and usability for use in hospitals.
\end{abstract}

\section{DESCRIPTORS}

Virtual Reality. Metabolic Syndrome Versus. Rehabilitation.

${ }^{1}$ Especialista em Atenção à Saúde Cardiometabólica do Adulto - HU/FURG. Programa de Residência Integrada Multiprofissional Hospitalar com Ênfase na Atenção à Saúde Cardiometabólica do Adulto (RIMHAS). Professora do Instituo Federal de Educação, Ciência e Tecnologia / Campus Pelotas.

${ }^{2}$ Doutor em Educação em Ciências (FURG). Professor Adjunto da Escola Superior de Educação Física - ESEF da Universidade Federal de Pelotas - UFPel. Pelotas, RS, Brasil.

${ }^{3}$ Educador Físico do Hospital Miguel Rieth Correa Jr. - HU/FURG/EBSERH. Mestre. Programa em Ciências da Saúde da Faculdade de Medicina - FURG. Preceptor da Residência Integrada Multiprofissional com Ênfase na Atenção à Saúde Cardiometabólica do Adulto (RIMHAS). 
A s doenças cardiometabólicas são uma das principais causas de morte no mundo ${ }^{1}$, podendo ser consideradas como a epidemia do século XXI. Os agravos como a hipertensão arterial, o diabetes mellitus e a obesidade também acarretam risco para as doenças cardiovasculares, assim como para acidente vascular cerebral ou o infarto agudo do miocárdio, de acordo com a Sociedade Brasileira de Endocrinologia e Metabologia².

Nesse sentido a Sociedade Brasileira de Diabetes $^{3}$ recomenda que a prevenção esteja intimamente relacionada ao tratamento de fatores de risco cardiometabólico, frequentemente associados ao diabetes mellitus, tais como: hipertensão arterial, dislipidemia, obesidade, tabagismo e sedentarismo.

Para a prevenção e controle destas doenças e agravos, são necessárias intervenções no comportamento e no estilo de vida das pessoas, principalmente com a inclusão de exercício físico regular e de uma alimentação equilibrada. Atualmente, existe uma vasta literatura ${ }^{4-6}$ corroborando que um aumento nos níveis de atividade física pode contribuir para diminuir os efeitos dos fatores de riscos cardiometabólicos.

Uma possibilidade de intervenção é a utilização da realidade virtual (RV) dos videogames de movimento como ferramenta auxiliar na promoção da saúde. Os videogames de movimento, active videogames ou exergames (EXGs) são tipos diferentes de console de videogame, os quais exigem um esforço físico maior quando comparado com os videogames tradicionais ${ }^{7}$. Assim, um EXG exige o trabalho de algumas capacidades físicas como a resistência, a coordenação motora, a velocidade, a força, o equilíbrio e a flexibilidade para suportar a jogabilidade e a narrativa dos games. A emulação perceptiva e de atuação dos EXGs, propiciada por mecanismos de RV e tecnologia de rastreio, permite essa interação dos movimentos do corpo com o jogo?.

A utilização da RV em determinados treinamentos tem demonstrado que pessoas engajadas nesses programas perdurariam no tratamento por mais tempo ${ }^{8}$. Assim, as inovações tecnológicas têm papel fundamental para o aperfeiçoamento, a qualidade e a diferenciação do tratamento de pacientes, onde sua aplicação torna mais dinâmica a rotina de exercícios $^{9}$. O olhar para este tipo de intervenção pode representar um aspecto importante no tratamento de pacientes, principalmente pelo fator motivacional que esta ferramenta possui em aderir o paciente ao programa.

O Uso de EXGs em programas de atividade física tem se mostrado eficaz, em crianças obesas ${ }^{10}$, por exemplo, e a sua utilização melhorou consideravelmente não apenas - $\mathrm{VO}_{2}$ máximo, como também promoveu uma redução na circunferência da cintura, além de aumentar o nível de atividade física e a percepção de um estilo de vida mais ativo. Entre mulheres adultas obesas ${ }^{11}$, um programa de exercícios utilizando EXGs promoveu uma redução dos triglicerídeos, colesterol e LDL, além disso, o seu uso também contribuiu para uma diminuição do IMC, massa corporal, circunferência abdominal e do percentual de gordura, reduzindo assim os riscos cardiovasculares nesta população.

Diante deste contexto é possível utilizar a realidade virtual dos EXGs como ferramenta em programas de reabilitação em hospitais? 
Sendo assim, o objetivo desta pesquisa foi investigar os efeitos de um programa de exercício físico com caminhada e EXG sobre as capacidades físicas (velocidade de movimentação, equilíbrio, força de preensão, capacidade aeróbica); variáveis hemodinâmicas (pressão arterial PA, saturação de oxigênio $\mathrm{SPO}_{2}$, frequência cardíaca $\mathrm{FC}$, taxa glicêmica); variáveis antropométricas (massa corporal, estatura, e circunferências da cintura, do quadril e do abdômen); qualidade de vida e percepção subjetiva de esforço (PSE), em pacientes cardiometabólicos submetidos ao treinamento com o EXG em um Hospital Universitário.

\section{METODOLOGIA}

O estudo se caracterizou como pesquisa experimental com um grupo intervenção, o qual foi submetido a uma rotina de exercícios aeróbicos e ao treinamento com a ferramenta EXG. As intervenções foram realizadas no Centro de Reabilitação Física e Cardiometabólica do Hospital Universitário Dr. Miguel Riet Correa Jr. (HU-FURG) na Cidade de Rio Grande, RS.

Após a aprovação do estudo pelo Comitê de Ética em Pesquisa na Área da Saúde (CEPAS - FURG) da Universidade Federal do Rio Grande - FURG sob o n ${ }^{\circ}$ 115/2017, 15 indivíduos adultos, com afecções cardiometabólicas (diabetes mellitus, hipertensão, insuficiência cardíaca, hipotireoidismo, dislipidemia) com idade superior a 18 anos e que fazem acompanhamento no Centro Integrado do Diabetes (CID/FURG), participaram da pesquisa. A amostra foi selecionada intencionalmente, de maneira a incluir na pesquisa to- dos os sujeitos que estivessem realizando um tratamento de reabilitação cardiometabólica no hospital durante a realização da pesquisa. Como critérios de inclusão foi estabelecido que estariam elegíveis para participar da pesquisa os indivíduos participantes do programa de reabilitação cardiometabólica do hospital que aceitassem, voluntariamente, participarem do tratamento alternativo.

Aos voluntários foi exposto a metodologia utilizada e como se daria o andamento das atividades, assim, antes das intervenções, todos assinaram o Termo de Consentimento Livre e Esclarecido. Inicialmente foi realizada a anamnese e a avaliação antropométrica, logo após, os indivíduos realizaram os testes de TC6, dinamometria manual, equilíbrio estático e também foi aplicado o questionário SF-36.

Para investigar o perfil dos voluntários, previamente a participação, foi realizada uma anamnese contendo o histórico pregresso de patologias, comorbidades, tratamento medicamentoso e hábitos alimentares. Os fatores de risco para doença arterial coronariana e avaliação de risco para $\mathrm{DCV}^{12}$ também foram avaliadas.

Como instrumentos de medidas pré e pós o período de treinamento foram utilizados o teste de equilíbrio estático ${ }^{13}$, o teste de

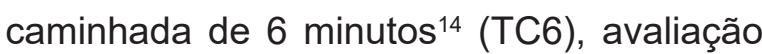
da força manual (Dinamometria). As medidas antropométricas de circunferência do quadril $(\mathrm{Cq})$, circunferência do abdômen (Ca), circunferência da cintura $(\mathrm{Cc})$, peso e altura também foram aferidas. Foi utilizado para mensuração da qualidade de vida o instrumento SF- $36^{15}$. A percepção subjetiva de esforço (PSE) foi avaliada por meio da escala adaptada de BORG $(0-10)^{25}$, a qual foi utilizada após a sessão de 
exercícios e também para mensuração de carga global da sessão.

Os materiais utilizados no presente trabalho foram: dinamômetro manual marca DayHome (modelo Y-55); uma trena antropométrica de fibra simples com trava marca Sanny com precisão de $0,1 \mathrm{~cm}$; um estetoscópio e esfigmomanômetro marca BIC; cardiofrequencímetro marca Polar modelo H10; oxímetro marca ChoiceMMed modelo MD300C1 Fingertip e glicosímetro marca Accu-Chek Performa. Além destes equipamentos, também foi utilizado para realizar a intervenção um console Xbox 360 com sensor Kinect e o game YourShape: Fitness Evolved (Ubisoft 2010) e um projetor multimídia padrão powerlite s/4,1800 lumens da marca Epson.

As sessões de treinamento tiveram duração de 60 minutos, três vezes por semana, perfazendo um total de 12 sessões. 0 treinamento foi iniciado com uma caminhada na esteira, durante 30 minutos e logo após os exercícios em ambiente virtual no EXG, também durante 30 minutos. A velocidade da caminhada foi individualizada para cada sujeito da pesquisa, sendo estipulada conforme resultado no TC6 e a velocidade de caminhada em esteira rolante adequada para que fosse mantida com PSE moderada (3 na escala de Borg adaptada). Os exercícios para o equilíbrio, para a postura e para os membros inferiores e superiores foram realizados através do game YourShape: Fitness Evolved, no modo de jogo Personal Trainer, o qual constituiu de 2 a 3 rotinas com média de 3 exercícios em cada rotina, 3 a 4 séries variando de 12 a 24 repetições, entre eles: corrida estacionária, agachamentos, movimentos de esquiva do Boxe e caminhada lateral, combinados com flexão e extensão ou adução e abdução de ombros e cotovelos. O game atribui pontuação de acordo com a precisão dos movimentos realizados, como ângulos de flexão e extensão e número de repetições.

Durante as sessões foram mensuradas as variáveis metabólicas PA, FC (pico e média), $\mathrm{SPO}_{2}$ e taxa glicêmica antes e após as sessões (pré e pós), além da quantificação da PSE após cada sessão. Além destes procedimentos, foi necessário realizar uma familiarização com o ambiente virtual e com os procedimentos de prática do EXG. Os participantes foram orientados verbalmente quanto aos procedimentos através de uma prévia explicação sobre o funcionamento do game, posicionamento em frente ao sensor de movimento e pontuação do game.

Com relação aos testes estatísticos, a normalidade dos dados foi verificada por meio do teste Shapiro-Wilk, os dados não paramétricos foram comparados através do teste de Wilcoxon comparando pré e pós. Os dados paramétricos foram apresentados em média e desvio padrão. Foi utilizado o teste $\mathrm{t}$ para comparação de dados pareados (pré e pós). A análise de variância ANOVA medidas repetidas foi utilizada para identificação das diferenças da variável pré e pós da $1^{\mathrm{a}}$ e $12^{\mathrm{a}}$ sessões. Para identificar os momentos que apresentaram diferenças foi utilizado o teste de Bonferroni. A taxa de erro alfa menor que $5 \%(p<0,05)$ foi considerada estatisticamente significativa.

\section{RESULTADOS}

A Tabela 1 descreve as variáveis antropométricas e as capacidades físicas investiga- 
Tabela 1. Média e desvio padrão dos valores relacionado às variáveis antropométricas e capacidades físicas no pré-teste $\left(1^{\circ}\right.$ sessão) e pós-teste $\left(12^{\circ}\right.$ sessão) após treinamento realizado com Exergame

\begin{tabular}{lccl}
\hline & $1^{\circ}$ sessão & $12^{\circ}$ sessão & $p$ \\
\hline IMC (ua) & \multicolumn{2}{c}{$\mathrm{n}=15$} & \\
Cc (cm) & $30,18 \pm 10,33$ & $30,4 \pm 9,86$ & 0.4942 \\
Ca (cm) & $96,73 \pm 19,46$ & $95,7 \pm 18,7$ & $0.0480^{*}$ \\
Cq (cm) & $103,3 \pm 22,3$ & $95,7 \pm 18,77$ & 0.3434 \\
RQC (ua) & $107,9 \pm 18,9$ & $106,6 \pm 17,2$ & 0.0637 \\
Dinamometria D (N) & $0,89 \pm 0,0$ & $0,89 \pm 0,74$ & 0.8874 \\
Dinamometria E (N) & $28,9 \pm 10,5$ & $30,09 \pm 10,16$ & 0.1744 \\
Eq est D (s) & $27,5 \pm 9,4$ & $26,61 \pm 8,29$ & 0.2109 \\
Eq est E (s) & $52,3 \pm 65,6$ & $38,4 \pm 36,90$ & 0.1927 \\
TC6 (m) & $36,7 \pm 97,7$ & $48,60 \pm 48,56$ & 0.0610 \\
Massa corporal $(\mathrm{kg})$ & $5,1 \pm 0,6$ & $5,42 \pm 0,63$ & 0.0855 \\
\hline
\end{tabular}

*Valores estatisticamente significativos para $p<0,05$

Tabela 2. Média e desvio padrão dos valores relacionado à percepção subjetiva de esforço de (PSE) e percentual acertos no game

\begin{tabular}{cccc}
\hline & $1^{\circ}$ sessão & $12^{\circ}$ sessão & $p$ \\
\hline \multicolumn{4}{c}{$\mathrm{n}=15$} \\
\hline PSE (ua) & $2,33 \pm 0,48$ & $2,93 \pm 0,79$ & $0,0025^{*}$ \\
$\%$ Game & $59,86 \pm 18,33$ & $55,93 \pm 23,32$ & 0,3931 \\
\hline
\end{tabular}

*diferença estatisticamente significativa para $p<0,05$

Tabela 3. Comparação dos valores de média e desvio padrão das variáveis hemodinâmicas, pressão arterial sistólica (PAS), pressão arterial diastólica (PAD, frequência cardíaca $(F C)$, glicose e saturação de oxigênio $\left(\mathrm{SpO}_{2}\right)$ entre a $1^{\circ}$ e $12^{\mathrm{a}}$ sessão após o uso do EXG.

\begin{tabular}{l|c|c|c|c|c}
\hline \multirow{2}{*}{} & \multicolumn{2}{|c|}{$1^{\circ}$ sessão } & \multicolumn{2}{c|}{$12^{\circ}$ sessão } & $p$ \\
\cline { 2 - 5 } & Pré & Pós & Pré & Pós & \\
\hline PAS (mmHg) & $119,33 \pm 16,67$ & $120,66 \pm 17,51$ & $116,67 \pm 17,18$ & $116 \pm 18,04$ & 0.6961 \\
PAD (mmHg) & $76 \pm 8,28$ & $74 \pm 7,36$ & $74,66 \pm 12,45$ & $75,33 \pm 10,0$ & 0.8783 \\
FC pico (bpm) & $79,86 \pm 11,42$ & $86,06 \pm 18,84^{*}$ & $82,86 \pm 13,7^{*} \dagger$ & $91,86 \pm 15,0^{*} \dagger \ddagger$ & 0.0329 \\
GGlicose (mg/dL) & $151,2 \pm 62,92$ & $124,87 \pm 45,49^{*}$ & $128,8 \pm 51,29^{*} \dagger$ & $108,73 \pm 31,48^{*} \dagger \ddagger$ & 0.0307 \\
$\mathrm{SpO}_{2}(\%)$ & $94,26 \pm 2,65$ & $94,2 \pm 2,33$ & $94,06 \pm 2,28$ & $94,53 \pm 32,0$ & 0.9324 \\
\hline
\end{tabular}

*diferenças estatisticamente significativas comparado com pré da $1^{\text {a }}$ sessão †diferenças estatisticamente significativas comparado com pós da $1^{\text {a }}$ sessão $\ddagger$ diferenças estatisticamente significativas comparado com pré da $12^{a}$ sessão 
das, podendo-se observar uma melhora nas variáveis relacionadas ao condicionamento cardiorrespiratório (TC6), força manual (Dinamometria) e equilíbrio (Eq est). Em relação às variáveis antropométricas, percebeu-se que ocorreu uma redução nas medidas de circunferência do quadril $(\mathrm{Cq})$, abdômen (Ca) e cintura (Cc). Porém, apenas a Cc apresentou diferença estatisticamente significativa $(p=0,048)$ devido ao de treinamento quando comparado os valores pré $(96,73 \pm 19,46)$ e pós $(95,66 \pm 18,77)$.

Conforme a Tabela 2, após 12 sessões de treinamento com EXG, os resultados indicam uma diferença estatística significativa para a PSE $(p=0,0025)$, percebendo-se um aumento da média na $1^{a}$ sessão $(2,33 \pm 0,48)$ em comparação a $12^{a}$ sessão $(2,93 \pm 0,79)$. A média do percentual de acerto no game ao longo do período de treinamento reduziu de $(59,86 \pm 18,33 \%)$ para $(55,93 \pm 23,32 \%)$ e não ocorreu diferença estatística ( $p=0,3931)$.

A Tabela 3 apresenta as variáveis metabólicas da pré e pós-sessão de treinamento com o uso do game, na $1^{\circ}$ e na $12^{\circ}$ sessão, percebeu-se que ao longo do período a FC pico e a taxa glicêmica apresentaram diferença significativa $(p=0,0329)$ e $(p=0,0307)$, respectivamente. Pode-se identificar por meio da Anova de duas vias pra medidas repetidas, seguida de post hoc de Bonferroni para $(p<0,05)$, que todos os momentos são diferentes tanto para variável FC quanto para a variável de taxa glicêmica.

Em relação à $\mathrm{FC}$ foram encontradas diferenças estatísticas entre o pré (repouso) e o pós-treinamento, em todas as sessões. Observou-se também uma diferença estatística entre o pré (repouso) da $1^{a}$ sessão $(79,86 \pm 11,42)$ e o pós-treino na $12^{\mathrm{a}}$ sessão $(91,86 \pm 15 ; p=0,0329)$, com o valor pós-treino mais elevado entre as sessões. Além disso o valor médio da FC pico nas sessões (145,8 8 $21,1 \mathrm{bpm})$ também apresentou diferença significativa ( $p<0.001$ ) quando comparado com o valor médio de repouso (88,4 $\pm 13,2$ bpm).

Também foi observada uma diferença significativa na média da taxa glicêmica de pós da $12^{\mathrm{a}}$ sessão $(108,73 \pm 31,48 ; p=0,0307)$ quando comparado com valores de pré da $1^{\mathrm{a}}$ sessão $(151,2 \pm 62,92 ; p=0,0307)$ e quando comparado ao pré da $12^{a}$ sessão $(128,8 \pm$ 51,29; $p=0,0307)$.

Em relação ao questionário SF-36 foi utilizado o Teste de Wilcoxon com $(p<0,05)$, comparando os resultados do pré e pós-treinamento com EXG. Foram encontradas diferenças estatisticamente significativas e com média dos valores acima de 50 no escore final para os aspectos da capacidade funcional $(p=0,0152)$, aspectos físicos $(p=0,007)$, percepção de dor $(p=0,011)$, vitalidade $(p=0,003)$ e saúde mental $(p=0,001)$, como pode ser observado nos gráficos das Figuras de 1 a 5 , caracterizando uma melhora no estado de saúde física, nesses componentes. Os aspectos relacionados ao estado geral de saúde $(p=0,401)$, aspectos sociais $(p=0,114)$ e aspectos emocionais $(p=0,179)$, como pode ser observado os valores de $p$, não apresentaram relevância significativa. 
Figura 1. Gráfico relacionado às questões sobre a capacidade funcional pelo SF-36



Figura 2. Gráfico relacionado às questões sobre limitação por aspecto físico pelo SF- 36



Figura 3. Gráfico relacionado ás questões sobre dor pelo SF-36

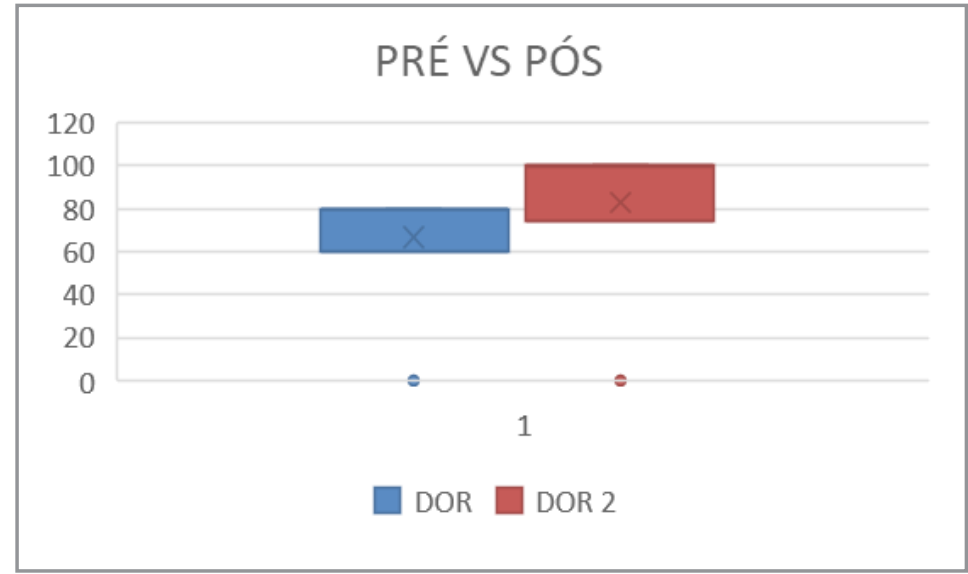


Figura 4: Gráfico relacionado às questões sobre vitalidade pelo SF-36

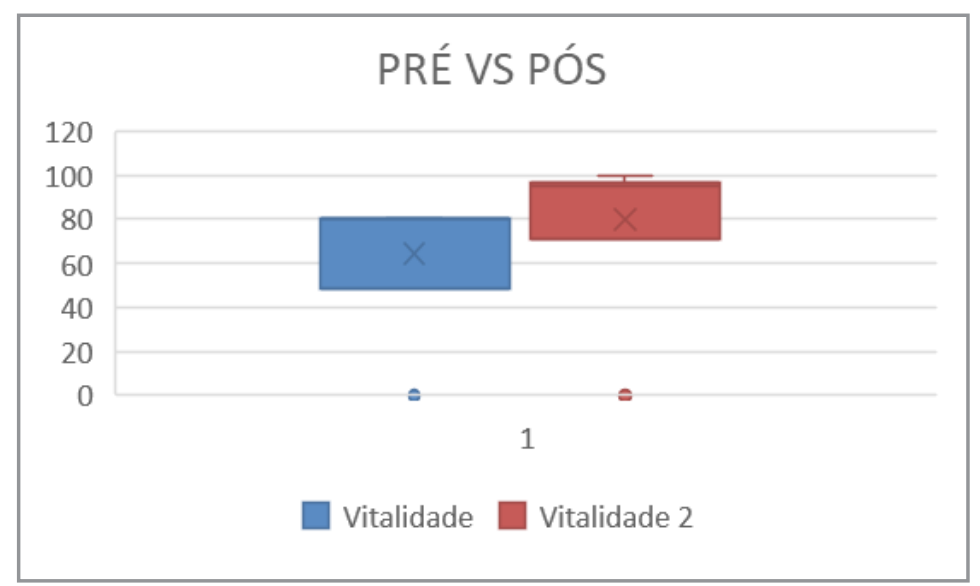

Figura 5: Gráfico relacionado as questões sobre saúde mental do SF-36

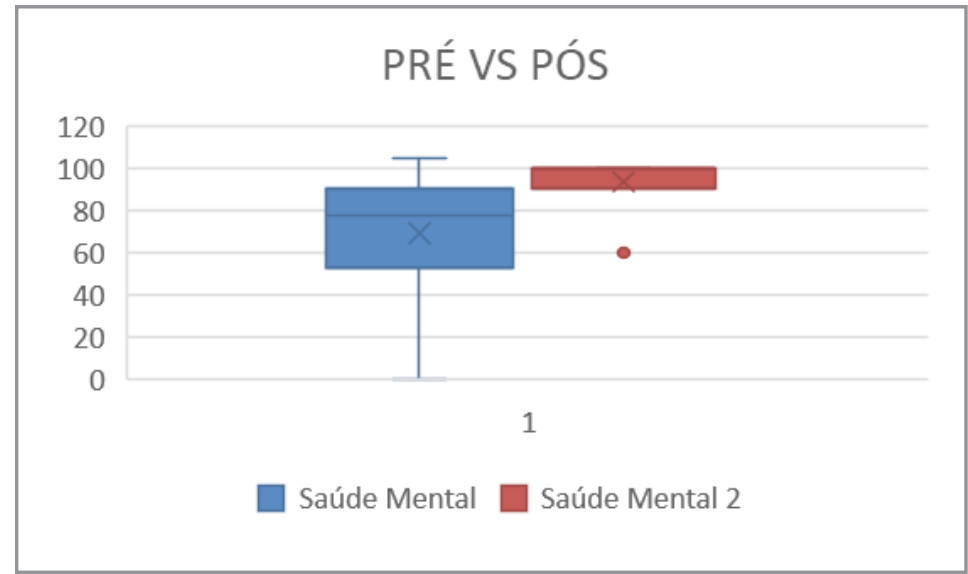

\section{DISCUSSÃO}

Este estudo tem um caráter inovador, visto que foi realizado dentro de um hospital, um local no qual a reabilitação física historicamente tem descartado o lúdico e onde os indivíduos necessitam de mecanismos motivadores para realização de exercício físico.

Nesta pesquisa foram encontradas diferenças estatisticamente significativas entre a primeira e a última sessão de treinamen- to, caminhada e EXG (YourShape: Fitness Evolved), para as variáveis Cc, PSE, taxa glicêmica e alguns aspectos do questionário de qualidade de vida como a capacidade funcional, os aspectos físicos, a dor, a vitalidade e a saúde mental. Além disso, a taxa glicêmica também apresentou uma diferença significativa entre o pré e o pós em todas as sessões, o que evidencia o potencial do EXG utilizado como ferramenta no controle desta variável. Em relação à FC foram encontradas 
diferenças estatísticas entre o pré (repouso) e o pós-treinamento, em todas as sessões e o valor de FC pico também apresentou diferença significativa quando comparado com o valor médio de repouso. Estes resultados evidenciaram o caráter inovador da RV, especialmente os EXGs, devido as suas características motivacionais e o seu potencial para a prescrição de exercício físico, assim como, o baixo custo e a usabilidade para uso em hospitais, podendo ser um instrumento que favoreça a adesão à prática de exercício físico até que o indivíduo atinja os níveis mínimos recomendados ${ }^{26}$.

A redução na taxa glicêmica e na $\mathrm{Cc}$ comprovam o esforço físico exigido no game, o qual foi capaz de produzir mudanças em um público com déficit cronotrópico, ressaltando a importância desse estudo ${ }^{25}$. Embora a sessão de treinamento com o EXG tenha sido realizada apenas em 30 minutos por sessão, combinado com 30 minutos de caminhada, estes resultados evidenciaram o potencial dos EXGs como ferramentas em programas de reabilitação em hospitais. Esses resultados corroboram com os valores obtidos na pesquisa de Amorim et al ${ }^{11}$., os quais investigaram o uso de EXGs em 12 mulheres com risco cardiovascular, os pesquisadores encontraram uma diminuição significativa para o índice de massa corporal $(10,5 \%)$, percentual de gordura $(10,9 \%)$, peso $(5,6 \%)$, circunferência abdominal $(2,3 \%)$, circunferência do quadril $(2,5 \%)$ e circunferência da cintura $(1,9 \%)$.

A inclusão de caminhada em esteira fez parte da estratégia deste estudo, visto que programas de reabilitação com esta população usualmente utilizam atividade aeróbia com exercícios resistidos ${ }^{25}$. O exercício aeróbio é recomendado como parte do tratamento de reabilitação em pacientes cardiometabólicos devendo ser incluso em qualquer programa de reabilitação, como o que ocorreu no presente estudo. Neste sentido, a intenção desta pesquisa foi substituir parte do treinamento de reabilitação, ou seja, substituir os exercícios resistidos em ambiente físico por exercícios resistidos em ambiente virtual.

Os sujeitos da pesquisa apresentaram respostas cronotrópicas comprometidas em um primeiro momento, sendo assim, a opção por uma estratégia simples e viável foi a utilização da PSE por meio da escala de BORG, focando na percepção de esforço e nas manifestações de sintomas, uma prática bastante recomendada ${ }^{25,26}$. Como o presente estudo procurou salientar os efeitos do treinamento com o EXG YourShape: Fitness Evolved, optou-se pela manutenção do volume e da intensidade do exercício em esteira permitindo que os efeitos do game se destacasse.

O game utilizado foi escolhido devido à similaridade dos exercícios exigidos no gameplay com os exercícios realizados nas sessões de reabilitação cardiometabólica. Além disso, o game Your Shape-Fitness Evolved foi citado como um dos melhores games em uma pesquisa que investigou diferentes sistemas para avaliação do controle postural ${ }^{16} \mathrm{em}$ indivíduos idosos, em decorrência da capacidade do Framework em avaliar se os exercícios exigidos estão sendo realizados com a angulação e a amplitude idêntica ao game.

Os resultados encontrados nesta pesquisa corroboram com uma pesquisa ${ }^{17}$ que investigou o uso de EXGs em mulheres com mais de 61 anos de idade pertencentes a um grupo de risco cardiovascular, no qual 
os efeitos do exercício realizado no EXG e em esteira apresentaram valores similares para a capacidade aeróbia. Na pesquisa citada anteriormente, os pesquisadores afirmam que o grupo que realizou exercícios com EXGs mostrou mais aderência ao programa devido à motivação gerada pelo game, além disso, o seu uso pode satisfazer as recomendações do ACSM e representa uma alternativa para o tratamento e a prevenção para risco cardiovascular.

Logo, o uso do game YourShape Fitness Evolved em indivíduos cardiometabólicos permitiu estimular o sistema cardiovascular de modo que o game promoveu uma alteração da PSE em todos avaliados, de leve para moderada e possibilitou a melhora do controle glicêmico. Corroborando com este estudo de Kempf \& Martin ${ }^{18}$, os quais analisaram variáveis metabólicas e antropométricas em idosos associados à prática de EXGs, os resultados apontaram uma melhora no quadro geral do público-alvo, possibilitando um aumento no nível de atividade física dos participantes após uma intervenção de 30 minutos diários durante 12 semanas, no qual uma diminuição significativa nos níveis de glicemia em jejum e da $\mathrm{HbA} 1 \mathrm{c}$ foram identificados.

Os resultados também indicaram um aumento significativo entre a $1^{\mathrm{a}} \mathrm{e}$ a $12^{\mathrm{a}}$ sessão para a PSE. Algumas possíveis explicações para este fato se relacionam com as fases do game. Conforme Vaghetti et al. ${ }^{19}$ um dos elementos mais importantes no gameplay para manter o jogador ao jogo são os desafios, ou seja, no ambiente virtual dos games o desafio é apresentado gradualmente ao jogador, no caso dos EXGs o desafio é apresentado na forma de intensidade do exercício ou nova tarefa motora. Desta forma, percebeu-se que os sujeitos relataram maior percepção de esforço do exercício conforme avançavam nas fases do game.

A força manual e o equilíbrio não sofreram modificações nos valores através dos protocolos investigados, provavelmente porque o game utilizado não exige trabalho de força manual em nenhum exercício proposto. Além disso, o game não apresenta exercícios específicos para treinamento de equilíbrio. Existem outros games com gameplay específico para trabalhar equilíbrio ${ }^{20-22}$, porém o uso do Your Shape Fitness Evolved nesta pesquisa se justifica pela similaridade com os exercícios já previamente utilizados em protocolos de reabilitação cardiometabólica.

Por meio do instrumento SF-36 foi observada uma diferença significativa nos componentes físicos quanto à capacidade funcional $(p=0,0152)$, às limitações por aspectos físicos $(p=0,007)$ e à percepção de dor $(p=0,011)$ dos indivíduos estudados. Essas diferenças significativas podem estar relacionadas ao aumento da PSE do game, pois o indivíduo pode ter se sentido desafiado e motivado no ambiente virtual a alcançar o objetivo que o game propôs, uma vez que o exercício em esteira teve sua PSE controlada. Quanto aos componentes mentais, também foi observado uma diferença significativa relativa à percepção de vitalidade $(p=0,003)$ e da saúde mental $(p=0,001)$. Estes valores podem estar relacionados também com os elementos do gameplay ${ }^{19}$, imersão e feedback. A imersão é um elemento capaz de levar o usuário para o ambiente virtual, proporcionando a sensação de estar em outro ambiente e a sensação de despreocupação consigo mesmo, enquanto o 
feedback relaciona-se com a aprendizagem. O sistema de feedback do game fornece ao usuário uma sensação de controle sobre sua própria performance.

Com relação aos componentes do questionário SF-36, Meneghini et al. ${ }^{23}$ também encontraram resultados significativos durante um programa com EXGs. Os pesquisadores relataram diferenças significativas para os aspectos psicológicos (autoestima, concentração, humor, raciocínio, memória e bem-estar), físicos (agilidade e condições físicas) e de interação social (troca de experiências, amizade e competitividade).

A proposta de introdução de EXG em programas de reabilitação deve ser considerada como um grande avanço na área da saúde, visto que, no atual mundo informatizado e digital é inevitável a participação no processo tecnológico e inegável que as ciências da saúde devem apropriar-se desses avanços, além dos aspectos motivacionais proporcionados por esta ferramenta.

Com este propósito, Artemisa e colaboradores ${ }^{24}$ salientam que existem inúmeras possibilidades de aplicação da RV a uma diversidade de domínios, de funções cognitivas, comportamentais, doenças neurológicas e incapacidades físicas. Com suas características inovadoras, a RV permite ultrapassar limitações das intervenções tradicionais, além do reconhecido potencial desta tecnologia, assistindo-se a um esforço crescente de validar a sua utilização na reabilitação em geral e, em particular, na reabilitação física.

\section{CONCLUSÃO}

Nesta pesquisa foram encontradas diferenças estatisticamente significativas entre a primeira e última sessão do treinamento de caminhada em esteira e EXG, para as variáveis taxa glicêmica, circunferência de cintura, percepção subjetiva de esforço e para alguns aspectos do questionário de qualidade de vida como a capacidade funcional, os aspectos físicos, a dor, a vitalidade e a saúde mental.

A substituição dos exercícios resistidos em ambiente físico por exercícios em ambiente virtual, por meio do exergame Your Shape Fitness Evolved permitiu ultrapassar as limitações das intervenções tradicionais, trazendo uma opção de baixo custo para uso em hospitais, aliado a sua facilidade para utilização, além dos fatores motivacionais que a imersão na realidade virtual proporciona. Percebe-se que para fins de treinamento físico o uso de Exergames evidencia a necessidade de avaliação das ações do usuário, identificando falhas, dificuldades e acertos que permitam observar o seu desempenho e o seu progresso ao longo do programa.

Embora ainda seja necessária uma maior investigação sem a associação de exercícios aeróbios e com novos protocolos e outros tipos de games, é viável a utilização de Exergames como ferramentas para reabilitação cardiometabólica em ambiente hospitalar. Destaca-se também que a implementação deste tipo de tecnologia no treinamento físico não vem com a ideia de substituir o papel do profissional de educação física, mas sim de ser propriamente uma ferramenta de reabilitação física. 


\section{REFERÊNCIAS}

1. Ferreira SRG, Chiavegatto Filho ADP, Lebrão ML, Duarte YADO, Laurenti R. Doenças cardiometabólicas. Rev Bras Epidemiol. 2019; 21(suppl2):1-13.

2. Faludi AA, Izar MCO, Saraiva JFK, Bianco HT, Chacra APM, Bertoluci MC, et. al. Diretriz brasileira baseada em evidências sobre prevenção de doenças cardiovasculares em pacientes com diabetes: posicionamento da Sociedade Brasileira de Diabetes (SBD), da Sociedade Brasileira de Cardiologia (SBC) e da Sociedade Brasileira de Endocrinologia e Metabologia (SBEM). Arq Bras Cardiol. 2017;109(6):1-45.

3. Milech A, Angelucci AP, Golbert A, Matheus A, Carrilho AJ, Ramalho AC. Diretrizes da sociedade brasileira de diabetes (2015-2016). São Paulo: AC Farmacêutica. 2016.

4. Lin X, Zhang X, Guo J, Roberts CK, McKenzie S, Wu W, Liu S, Song Y. Effects of Exercise Training on Cardiorespiratory Fitness and Biomarkers of Cardiometabolic Health: A Systematic Review and Meta-Analysis of Randomized Controlled Trials. J Am Heart Assoc. 2015; 4(7):1-28.

5. Campos ALP, Corrêa LQ, Silva MC, Rombaldi AJ, Afonso MR. Efeitos de um programa de exercícios físicos em mulheres hipertensas medicamentadas. Rev Bras Hipertens. 2009; 16(4):205-9.

6. Cruz PWS, Cardoso Jr CG, Cucato GG, Vancea DMM, Ritti-Dias RM. Perfil de risco cardiovascular em praticantes de exercícios físicos supervisionados. ConScientiae Saúde, 2011;10(3):460-466.

7. Vaghetti CAO, Ferreira ET, Cavalli AS, Monteiro-Junior RS, Del Vecchio FB. Exergames e sua utilização no currículo escolar: uma revisão sistemática. ConScientiae Saúde. 2017; 16(2):293-301.

8. Monteiro-Junior RS, Carvalho RJP, Silva EB, Bastos FG. Efeito da reabilitação virtual em diferentes tipos de tratamento. Rev Bras Ciên Saúde. 2011; 9(29):56-63.

9. Fernandes, FG. Santos SC, Oliveira LC, Rodrigues ML, Vita SSBV. Realidade virtual e aumentada aplicada em reabilitação fisioterapêutica utilizando o sensor Kinect e dispositivos móveis. Anais XII CEEL, Universidade Federal de Uberlândia - UFU; 13-17 de outubro 2014; Uberlândia, Brasil. 2014

10. Lamboglia CM, Silva VT, Vasconcelos Filho JE, Pinheiro $\mathrm{MH}$, Munguba MC, Silva Jr FV, Paula FA, Silva CA. Exergaming as a strategic tool in the fight against childhood obesity: a systematic review. J Obes. 2013;1-8.

11. Amorim MGS, Oliveira MD, Soares DS, Borges LS, Dermargos A, Hatanaka E. Effects of exergaming on cardiovascular risk factors and adipokine levels in women. J Physiol Sci. 2018; 68(5):671-8.

12. Chodzko-Zajko WJ, Proctor DN, Singh MAF, Minson CT, Nigg CR, Salem GJ, Skinner JS. Exercise and physical activity for older adults. Med Sci Sports Exer. 2009:41(7):1510-1530

13. Carnaval PE. Medidas e avaliação em ciências do esporte. Rio de Janeiro: Sprint, 1995.

14. ATS Committee on Proficiency Standards for Clinical Pulmonary Function Laboratories. ATS Statement: Guide- lines for the six-minute walk test. Am J Respir Crit Care Med. 2002; 166(1):111-17.

15. Ciconelli RM, Ferraz MB, Santos W, Meinão I, Quaresma MR. Brazilian portuguese version of the SF-36, a reliable and valid quality of life outcome measure. Rev Bras Reumatol. 1999; 39(3):143-150.

16. Tahmosybayata R, Bakera K, Godfreyb A, Caplana N Barrya G. Movements of older adults during exergaming interventions that are associated with the Systems Framework for Postural Control: A systematic review. Maturitas. 2018; 111:90-99.

17. Eun-Ah J, Shan-Shan W, Hyung-Rae H, Jung-Jun P, Saejong $\mathrm{P}$, Kyoung-Im C. Effects of exergaming in postmenopausal women with high cardiovascular risk: A randomized controlled trial. Clin Cardiol. 2020; 43(4):363-370.

18. Kempf K, Martin S. Autonomous exercise game use improves metabolic control and quality of life in type 2 diabetes patients - a randomized controlled trial. BMC Endocr Disord. 2013;13(57):1-9.

19. Vaghetti CAO, Monteiro-Junior RS, Finco MD, Reategui E, Botelho SSC. Exergames Experience in Physical Education: A Review. Phys Cult Sport Stud Res. 2018; 78:23-32.

20. Bieryla Ka, Dold NM. Feasibility of Wii Fit training to improve clinical measures of balance in older adults. Clin Interv Aging. 2013; 8:775-781.

21. Wüest S, Borghese NA, Pirovano M, Mainetti, R, Van De Langenberg R, De Bruin ED. Usability and Effects of an Exergame-Based Balance Training Program. Games Health J. 2014; 3(2):106-111114.

22. Mhatre PV, Vilares I, Stibb SM, Albert MV, Pickering L, Marciniak CM, Kording K, Toledo S. Wii fit balance board playing improves balance and gait in Parkinson disease. J Am Phys Med. 2013; 5:769-777.

23. Meneghini V, Barbosa AR, Mello ALSF, Bonetti A, Guimarães AV. Percepção de adultos mais velhos quanto à participação em programa de exercício físico com exergames: estudo qualitativo. Ciên Saúde Colet. 2016; 21(4):1033-1041.

24. Artemisa RD, Barbosa F, Marques A, Carvalho IP, De Sousa L, Castro-Caldas A. Realidade Virtual na Reabilitação: Por Que Sim e Por Que Não? Uma Revisão Sistemática. Acta Med Port. Nov-Dec. 2012; 25(6):414-421.

25. Carvalho $T$, Milani M, Ferraz AS, Silveira AD, Herdy AH, Hossri CAC, et al. Diretriz Brasileira de Reabilitação Cardiovascular - 2020. Arq Bras Cardiol. 2020; 114(5):943-987.

26. Ding D. Surveillance of global physical activity: progress evidence, and future directions. Lancet Glob Health. 2018 6(10):e1046-e7.

\section{CORRESPONDÊNCIA}

Karina Langone Vieira

Avenida Santos Dumont 653, Junção, Rio Grande.

E-mail: karinavieira_35@hotmail.com 